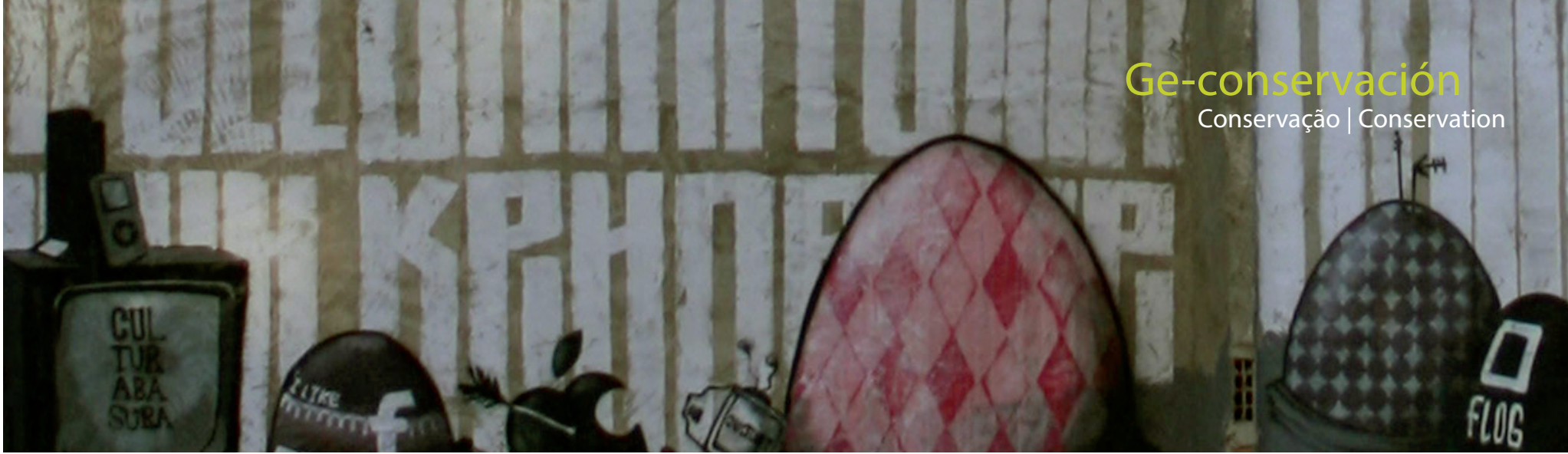

\title{
Entre la teoría de la restauración y el arte urbano, una paradoja disciplinar
}

\author{
Ana Lizeth Mata Delgado
}

Resumen: Este texto analizará de qué manera se han llevado a cabo las intervenciones de conservación-restauración actuales para el Arte Urbano, pues si bien nos enfrentamos a un tipo de producción artística efímera y procesual por naturaleza, hemos encontrado diversas obras que han sido protegidas, conservadas e incluso restauradas en algunos casos, bajo los preceptos teóricos de la Restauración.

La disciplina de la Conservación-restauración, se rige bajo los principios éticos y teóricos para establecer los límites y alcances de los procesos de restauración directa aplicados a un objeto al momento de llevar a cabo la intervención. Si bien desde el s. XIX diversos teóricos han establecido posturas específicas (e incluso contradictorias entre sí) en torno al establecimiento de estos principios, la mayoría coinciden en priorizar la materia y la trascendencia del bien en cuestión. En inicio se revisará cada uno de los temas por separado para dar paso al análisis de algunos casos que han sido intervenidos y de qué manera.

Entonces, en el caso del arte urbano ¿Es posible aplicar estos principios? ¿Cómo se establecen los criterios de intervención? ¿Es mera coincidencia su aplicación en la conservación-restauración del arte urbano?

Palabras clave: teoría de la restauración, arte urbano, conservación-restauración, criterios de intervención, análisis crítico

\section{Among the theory of restoration and the urban art, a discipline paradox}

Abstract: This text will discuss how interventions of conservation and restoration current for Urban Art, because although we face a kind of ephemeral and processual artistic production by nature, we found several works that have been protected, preserved and even restored in some cases under the theoretical precepts of the Restoration.

The discipline of conservation and restoration is governed by the ethical and theoretical principles to establish the limits and scope of direct restoration processes applied to an object when carrying out the intervention. Although from the s. XIX various theorists have established specific (and even contradictory) about the establishment of these principles, most agree prioritize matter and significance of the property in question.

Then, in the case of urban art: Is it possible to apply these principles? How the intervention criteria established? Is application in the conservation and restoration of urban art?

Key words: restoration theory, urban art, conservation and restoration, intervention criteria, critical analysis

\section{Introducción}

Actualmente se cuestiona cada vez más la relación (si es que existe) entre la Teoría de la Restauración y el Arte Urbano a través de la conservación-restauración de este último. A partir del siglo XVIII se comenzaron a establecer lineamientos concretos para regir una disciplina que es mucho más antigua. Si bien en sus inicios estos se enfocaron principalmente en los bienes arquitectónicos y arqueológicos, su aplicación y uso se ha ido modificando de manera que abarque un sin número de objetos, estableciendo la división global entre bien mueble y bien inmueble por destino, a este último corresponde el Arte Urbano. Si bien éstos principios de manera general, priorizan la permanencia del objeto y establecen hasta dónde y de qué manera es correcto y factible llevar a cabo la conservación - restauración de una obra, considerando incluso las diferencias existentes tanto en contexto donde 
se encuentra, el uso y función que tiene dentro de éste y los alcances e impacto que tendrá nuestra intervención sobre la piezas. En este caso, el impacto de nuestra acción no solo ser verá en el objeto como tal desde el punto de vista material, sino en la manera en como lo percibirá la comunidad que convive con este tipo de obras, dado que al momento en que se considera una intervención hay una implicación de reconocimiento de valores y resignificación de la obra que le otorga nuevas características e implicaciones para su conservación y comprensión por parte del espectador.

Como lo explica Philipot: "Lo que distingue a la restauración de cualquier reparación es que se dirige a una obra de arte. Sin embargo ese reconocimiento de la obra de arte, que funda a la restauración, es claramente un momento actual, que pertenece al presente histórico del espectador-receptor. La obra de arte no deja por ello de ser reconocida como producto de una actividad humana en un tiempo dado y en un lugar dado, y por lo tanto, como un documento histórico, como un momento de pasado. Al estar presente en la experiencia actual que la reconoce como tal, la obra no puede por lo tanto ser únicamente el objeto de un conocimiento científico histórico: forma parte integrante de nuestro presente vivido, dentro de una tradición artística que nos une a ella, y permite sentirla como una interpelación del pasado dentro de nuestro presente: una voz actual en la cual resuena ese pasado." (Philipot 2015:20)

En este caso si bien el autor hace referencia a la obra de arte, es importante rescatar el enunciado que hace habla sobre el momento actual, el cual pertenece al presente histórico de quien genera y percibe la obra en cuestión por tanto, si bien el Arte Urbano no se ha considerado como tal una obra de arte en estricto sentido, sí tiene un reconocimiento social que da paso en ciertos casos a ser sujeto de una intervención, pues ya no solo es la visión del artista la que se tiene que considerar, sino también a ese espectador que vive, convive y de cierta manera consume la obra; más adelante se analizarán una serie de casos en donde se plantea la intervención y con qué objeto.

\section{Conservar Arte Urbano, ¿es posible?}

De cierta manera, la presencia de la Teoría de la Restauración, estructura la norma y ésta a su vez condiciona y establece el rigor que deberá seguirse para acercarnos al sujeto de estudio desde cualquiera de nuestras perspectivas disciplinares. Sin embargo, cuando se plantea aplicar esta teoría a las producciones del siglo XX y XXI se comienza a analizar de manera distinta su devenir y alcance, pues si bien, existe claridad de qué manera se establecen estas normas aún en arte moderno, cuando empezamos a hablar de arte contemporáneo el planteamiento teórico se tambalea dado que existen una serie de producciones que ponderan la experimentación, la creatividad, la innovación, la mezcla de materiales incompatibles entre sí, no necesariamente buscan la trascendencia e incluso en algunos casos, estas producciones ni siquiera contemplan la perdurabilidad ni la permanencia para futuras generaciones, sino que se centran principalmente en el momento presente. Si evaluar estas producciones artísticas en algunos casos resulta complejo, cuando se habla de Arte Urbano se vuelve prácticamente imposible encontrar un punto de comunión, un análisis siquiera posible de cómo y para qué conservarlo, pero sobre todo surge como primer cuestionamiento el por qué.

Si bien la aplicación de la Teoría de la Restauración en obras como éstas resulta contradictorio pues se trata de un tipo de producción efímera, procesual y contestataria que en muchos casos es ilegal (espontánea) o que no tiene por intención ser conservada ni restaurada (o no en la mayoría de los casos), aunado a que no considera el trascender y/o perdurar como lo haría una pintura mural por ejemplo. Estaríamos atentando de cierta manera contra sus características primarias ya por el simple hecho de considerar conservarla y hacerla trascender.

Es importante analizar cuáles son las principales características del Arte Urbano, para comprender la importancia y el impacto que tendrá nuestra intervención, por ello podemos enunciar varias: este tipo de arte plantea un reclamo del espacio público, una reapropiación de las calles, generar un vínculo hacia afuera de los espacios expositivos y hacerse presente en un contexto alternativo. Por tanto este tipo de obras son transgresoras, su producción está muy ligada al activismo y no consideran en inicio la permanencia pues al ser generalmente de producción ilegal su tiempo de vida es limitado. Además es justo ese carácter sorpresivo y de apropiación del espacio. "En ocasiones se trata de trabajos planeados y materializados con elementos que están ligados a la localización, historia o caracterización social de sitios específicos, o desde luego, a las fantasías propias de los autores" (Arroyo 2015:276).

Por su parte, el Arte Urbano es considerado como una derivación del Street Art pues generalmente se refiere a aquellas producciones artísticas ubicadas en el espacio público producto de comisiones y/o festivales patrocinados, aunque hay casos en donde se integran también producciones ilegales y/o espontáneas. En muchos casos buscan transmitir mensajes directos que no solo se queden en el contexto callejero sino que trascienden hacia otros foros. "Muchos de estos mensajes quedan referidos como una denuncia, que en el momento en que fue realizada tal vez hubiese querido ser un fuerte grito de inconformidad" (Marcial 2012:310)

Partiendo de estas prerrogativas, quizá hablar de arte urbano y conservación-restauración dentro de un mismo enunciado resulta paradójico y cuestionable, ¿quién decide qué y cómo debe de conservarse? ¿Se hace una acción directa o solo se documenta? ¿Quién lo hace, un 
restaurador o el artista? Estas y otras tantas preguntas nos surgen al momento de siquiera considerar conservarlas.

\section{Principales factores de deterioro que determinan una posible conservación}

Antes de dar paso a la revisión de casos en donde las obras de Arte Urbano han sido intervenidas, vale la pena revisar cuáles son los factores de deterioro más comunes a los que están expuestas este tipo de manifestaciones artísticas y que por ende dan paso a la consideración de conservarlas y/o restaurarlas, aunado a la relevancia que tienen los artistas que la crearon. Se dará un panorama general de los deterioros vinculados al arte urbano para contextualizar la motivación que da paso una intervención, pues si bien estos dependen también del contexto y la técnica de manufactura con la que fueron realizados, existen una serie de deterioros comunes.

Uno de los deterioros más frecuentes es el vandalismo. Es curioso cómo dentro de la misma dinámica procesual y de creación, algunos artistas consideran que el trabajo de sus colegas no es digno de apreciarse al punto de que se "pisan" entre sí como una manera de demostrar su desacuerdo con la obra en cuestión. De acuerdo con la RAE, el vandalismo es cuando emerge el "Espíritu de destrucción que no respeta cosa alguna, sagrada ni profana." $Y$ es que estas acciones de cierta manera destruyen la obra materia y visualmente.

Estos actos no se limitan a la invasión con pintura o algún otro material (pinturas, aerosol, stickers, incisiones, manchas de pintura, entre otros) a fin de destruir o alterar la imagen, a veces llevan a cabo incisiones, rayones e incluso desprendimientos de la obra a fin de que desaparezca. [Figuras 1 y 2]

La intemperización de los materiales constitutivos. Este es un factor de deterioro inminente en el contexto callejero pues el impacto que tienen los agentes medioambientales sobre este

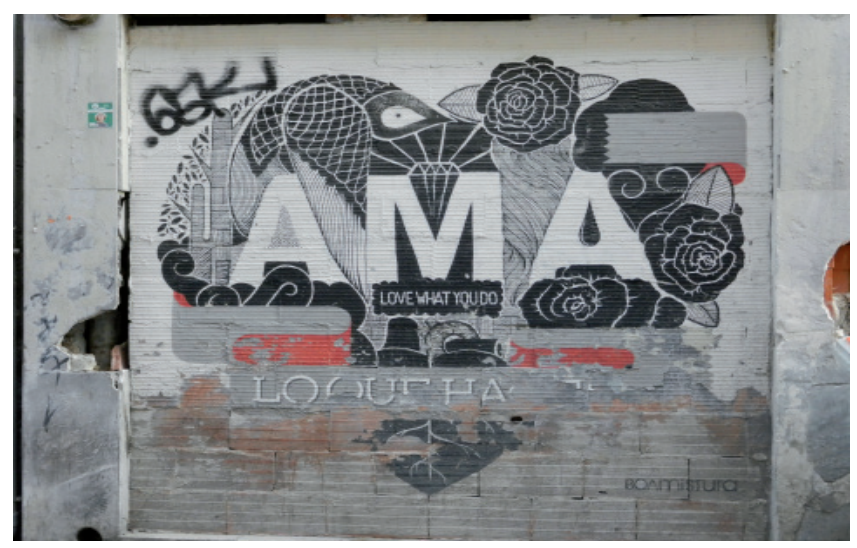

Figura 1. Graffiti sobre la obra de BoaMistura, (Madrid). Fotografía Ana Lizeth Mata Delgado.

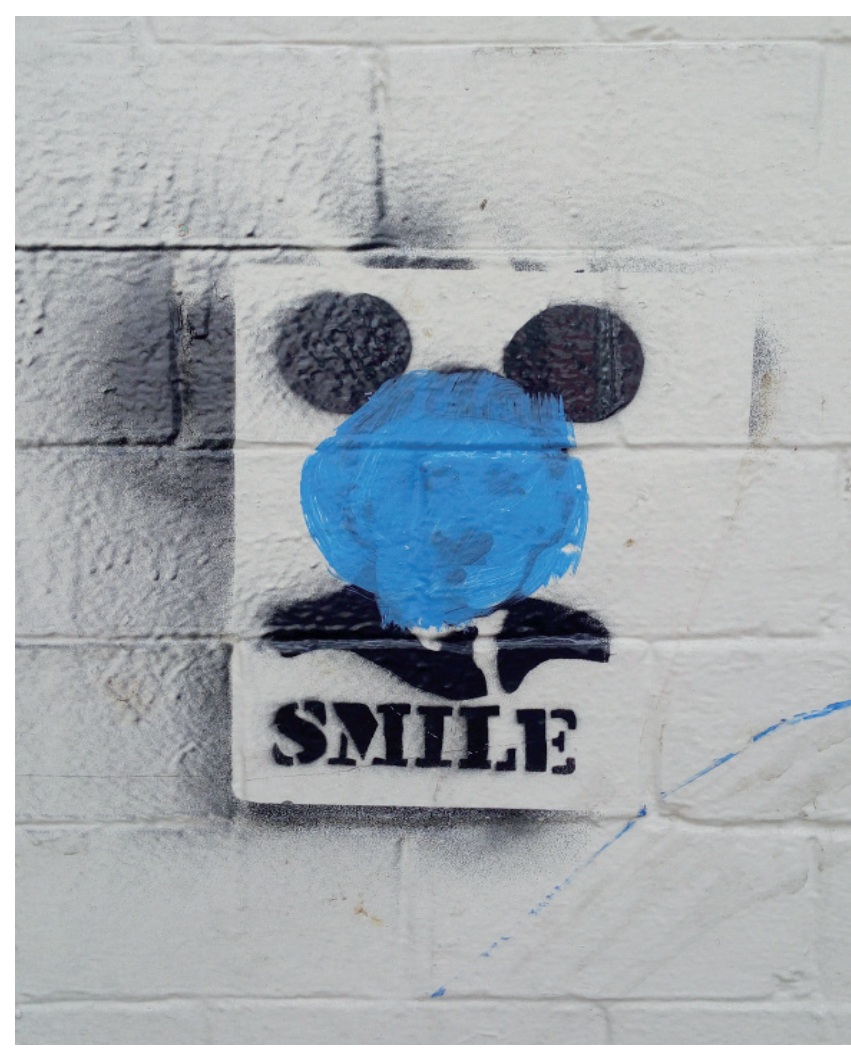

Figura 2. Colocación de pintura sobre stencil, artista desconocido, (CDMX). Fotografía Ana Lizeth Mata Delgado.

tipo de obras es constante, agresivo y genera daños que en otro tipo de contexto (espacios cerrados, museos, galerías, etc.) no se consideran. Deterioros como decoloración, escamación de capas pictóricas, abrasión, grietas, craqueladuras, pulverulencia, pérdida de adhesividad (en el caso de los stickers), manchas y desprendimientos son algunos de los deterioros que encontraremos en este tipo de obras y que en cualquier otro tipo de creación artística (a menos que lo consideren como un factor estético) sería factible de tratar por un profesional en el campo de la conservación. [Figuras 3 y 4 ]

Los desprendimientos y/o arranques de las obras de su contexto original; cada vez más se convierten en un método recurrente en los cuales se expolia la pieza para ser vendida o llevada a un contexto de galería o museo para el cual no fue creada. Es importante aclarar que si bien no aplica en todos los casos, cada vez más se observa esta acción como una manera de "conservar" la obra, descontextualizándola para ser insertada en un ambiente ajeno que termina por musealizar el objeto. Lo más grave es que en la mayoría de los casos el autor no está enterado y/o no ha dado su consentimiento.

Ejemplo de esto, fue el escándalo que se desató en 2013 por el arranque de la obra de Bansky ubicado en la calle principal de Wood Green, en el noroeste de Londres, el 14 de mayo de 2012, la obra titulada Slave Labour la cual hacía una crítica de la esclavitud infantil. Esta obra generó tal expectativa y apropiación por parte de la comunidad que incluso a la salida del metro se pusieron indicadores 


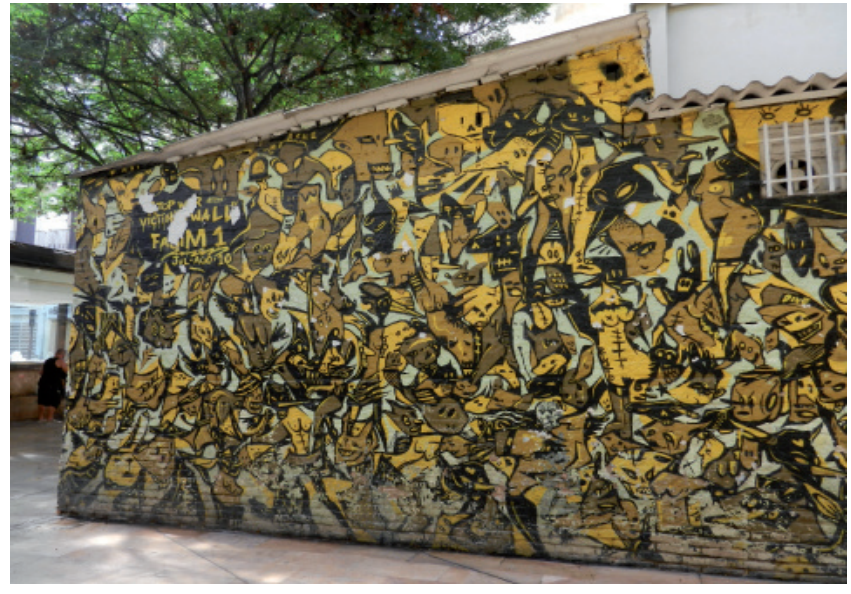

Figura 3. Abrasión y pérdida de capa pictórica. FASIM 1 (Valencia, España). Fotografía Ana Lizeth Mata Delgado.

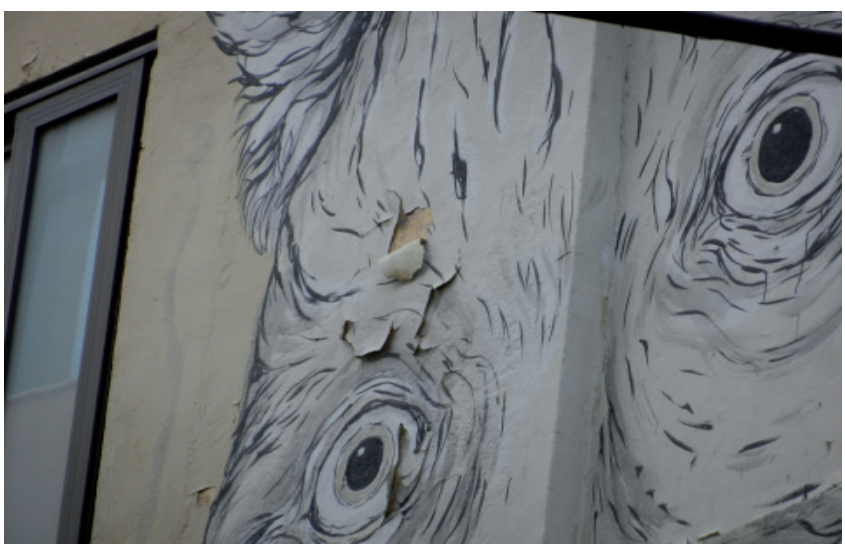

Figura 4. Detalle de escamación de capa pictórica, (Valencia, España). Fotografía Ana Lizeth Mata Delgado.

para que la gente supiera hacia dónde se encontraba y el transeúnte pudiera visitarla. Sin embargo, en 2013 esta obra fue arrancada sin ningún tipo de permiso, dando paso a un fuerte controversia pues horas después fue localizada en el catálogo de la casa de subastas Miami Fine Art Auctions para ser vendida. Este caso refleja claramente el interés no solo en la propuesta artística y el discurso planteado por el artista, también refiere al provecho que buscan extraer algunas personas con la venta de este tipo de obras, generalmente sin la autorización del artista. [Fig. 5]

Lo cuestionable en estos casos en sin duda la manera de eliminar y/o desaparecer una obra del contexto público, pues si bien está al alcance de todos, no significa que alguien tenga el derecho de sustraerla para beneficio personal y sin consultar ni considerar al creador.

\section{Alternativas de conservación}

A continuación se presentarán una serie de casos que ejemplifican las distintas alternativas con las que han sido intervenidos, lo interesante aquí es por quién, para qué y cómo han sido conservados. Partamos de una categorización previa de los alcances de estas intervenciones equiparando de cierta manera los principios de la Teoría de la Restauración y los alcances que ésta puede tener sobre el arte urbano.

Se pueden considerar tres momentos: documentación, conservación preventiva que podría equipararse a la mínima intervención necesaria y restauración. Finalmente en algunos casos se ha considerado también la reproducción de las obras cuando se tiene la documentación e información necesaria para llevarlo a cabo.

Una de las acciones de conservación más recurrentes desde los inicios de este movimiento artístico es la documentación, la cual se mantiene hasta ahora, cabe señalar que ésta no necesariamente la llevan a cabo profesionales en el campo de la conservación, sino desde sus inicios el graffiti neoyorquino comienza realizando fotografía de la imagen casi sin ningún tipo de texto explicativo. Actualmente existen textos especializados
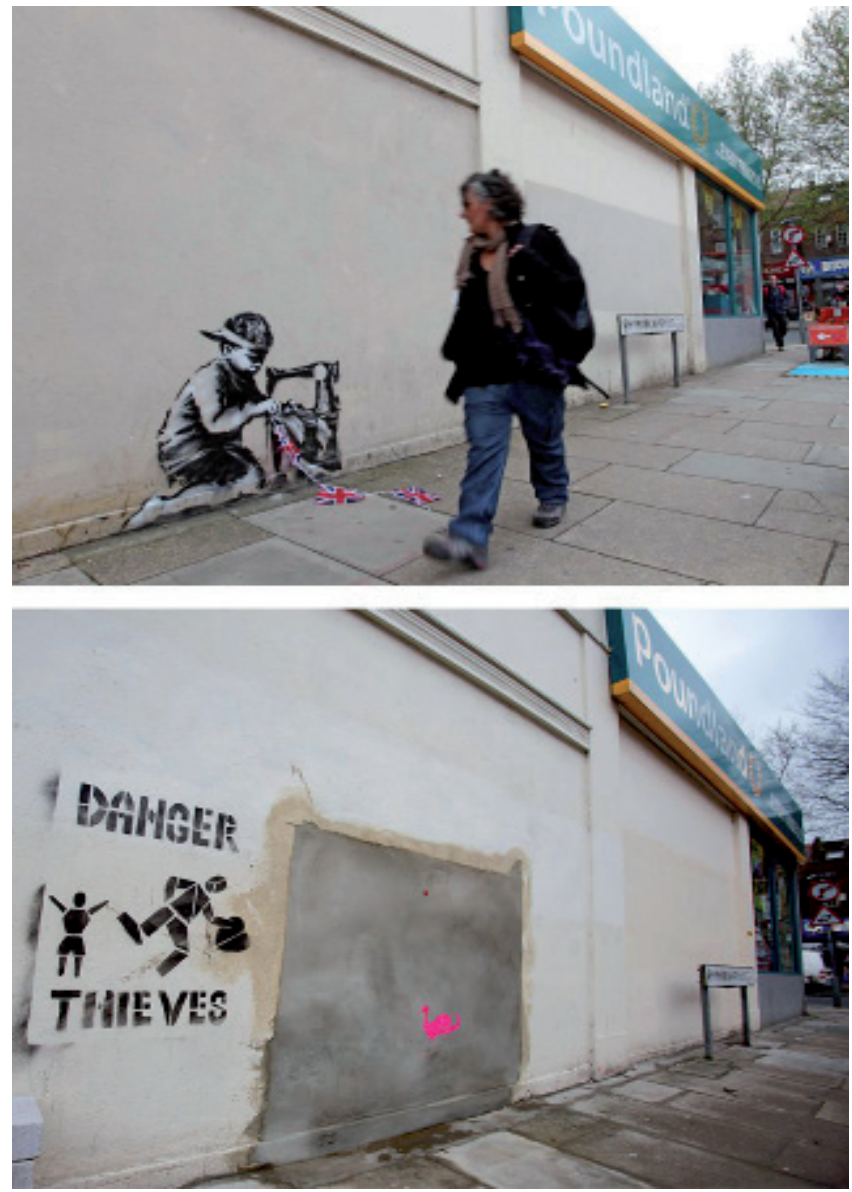

Figura 5. Banksy, Slave Labour. Wood Green, Haringey, Londres. Mayo de 2012 y su desaparición en 2013. Fotografía tomada de: http://www.elconfidencial.com/cultura/2013-06-01/londres-selevanta-en-armas-contra-la-venta-de-su-banksy_735833/ 
que no solo se basan en la imagen capturada sino que se trata de publicaciones especializadas en su investigación, sin embargo, desde la aparición de los teléfonos móviles inteligentes y otros dispositivos digitales como son las tablets, ipads, las cámaras de fotografía digital, por mencionar algunos, no solo es el artista o estudiosos del tema los que llevan a cabo el registro, sino el espectador, el ciudadano de a pie que se encuentra por casualidad y al que le resulta interesante, atractiva, curiosa, etc. una obra, acto seguido lleva a cabo un registro que compare en las redes sociales, nutriendo las páginas web de los creadores, compartiendo y difundiendo una obra de arte urbano, tenga o no la intención de ser conservada y/o restaurada materialmente. Esta manera de trascender es más sencilla, fácil y sobre todo masiva, actualmente existe millones de plataformas en la red que abordan a este tipo de producciones artísticas (Street Art, Graffiti, Arte Urbano, entre otros) las cuales siguen creciendo cada minuto.

La documentación se centra primordialmente en el registro fotográfico, pero también se puede considerar aquellas iniciativas que plantean cartografías de localización en donde se pueda ubicar la obra, su ubicación y una fotografía, tal es el caso de iniciativas como Street Art Chilango en la Ciudad de México o Madrid Street Art Project, por mencionar algunos. "Muchos de esos sitios no se limitan al hecho de documentar, sino además ofrecen recorridos virtuales o de forma presencial, usando los geolocalizadores para compartir la ubicación de las obras. En esos mapas suele mostrarse información sobre el artista y la ubicación, además de una imagen general de referencia." (Mata 2016:s/p)

Cabe mencionar que a pesar de que este fenómeno mediático que ha cobrado fuerza estos últimos años, la documentación de Graffitti como tal comenzó desde los años ochenta, pues fotógrafos como Martha Cooper y Henry Chalfant elaboraron un libro fundamental para el conocimiento y comprensión de éste titulado Subway Art, de 1984, considerado uno de los mayores exponentes publicados sobre este tema, con este texto se da inicio a una serie de documentos escritos y digitales enfocados al estudio de estas manifestaciones. A la fecha se han publicado un sinfín de textos especializados en el tema, lo que habla de un interés no solo por su difusión sino por su investigación, que a su vez ha servido para que sea asumido y comprendido como un fenómeno artístico actual que se desarrolla de manera paralela y del cual aún existen cuestionamientos sobre si debe o no conservarse, cómo y para qué o para quién.

La segunda categorización en la cual podemos hablar de una conservación preventiva en muchos casos está enfocada solo a la mera remoción de material ajeno a la obra para evitar su deterioro y promover su permanencia, en algunos caso se usan brochas, esponjas, o algún otro tipo de materiales de limpieza sin llevar a cabo una limpieza y/o intervención más profunda. Cabe señalar que ese tipo de intervenciones no solo se han registrado por parte de

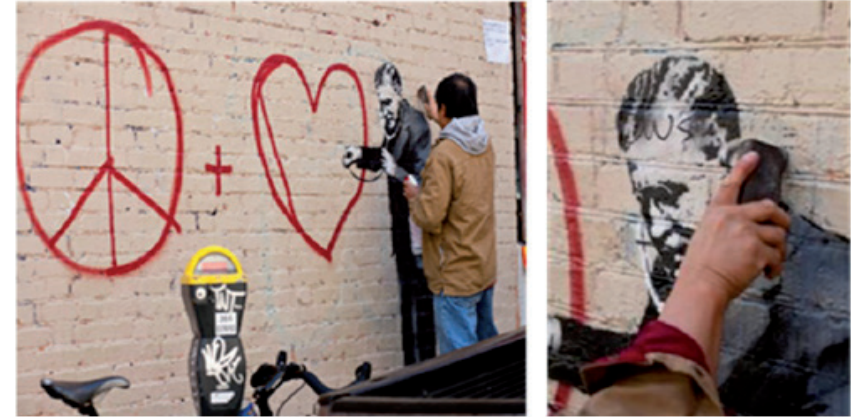

Figura 6. Limpieza de graffitis sobre la obra Peaceful Hearts de Banksy (Chinatown, San Francisco, CA). Fotografía D_Serna aka Tweak SF, tomada de http://warholian.com/2010/04/banksy-defaced-in-chinatown-warholian-san-francisco/

los conservadores sino en algunos casos son realizados por la comunidad, quien a fin de mantener estable la obra lleva a cabo estas labores. Esto nos habla ya de un reconocimiento y apropiación, sin embargo, la conciencia de aplicar un proceso basado en la mínima intervención necesaria, no está presente en sentido estricto. [Fig. 6]

Otra de las estrategias de conservación preventiva más recurrentes de conservación es la colocación de una placa de plexiglás o policarbonato al frente de la obra, atornillada al muro que contiene la obra.

Esto con la intención de que no la "pisen" otros artistas, la borren o sea dañada por los agentes medioambientales. Algunos de los artistas cuyas obras se han buscado conservar son primordialmente aquellos que representan un interés económico y de relevancia por su propuesta artística, como Blek le Rat y Banksy por mencionar algunos, pues más allá de la opinión de los creadores, son las personas cercanas y vinculadas a sus obras las que por conocimiento de su trabajo, el valor económico de las mismas o simplemente por un gusto, buscan protegerlas a fin de que se mantengan en las mejores condiciones posibles. [Fig. 7]

Esta acción de conservación se ha puesto sobre la mesa para ser cuestionada, dado que al momento de agregar un material ajeno a la obra e incluso al contexto, se hace referencia a dos cosas primordialmente: en primer lugar, al posible daño que pueda generarse por el microclima del entre el muro y el material plástico, además del daño a la estructura del muro que contiene a la obra como consecuencia de taladrar para sujetar el acrílico, y en segundo lugar, a la nueva visibilidad que se le da a la obra en cuestión, pues no sólo será percibida de forma distinta por el espectador, sino que se convierte en un punto focal para determinar que esa obra es valiosa y por tanto puede ser factible de robo o arranque en el peor de los casos. Esta tipo de actividad debe ser analizada si es necesaria antes de ser aplicada.

En el caso de obras que han sido restauradas podemos considerar un caso representativo en la obra de Black Le Rat, 


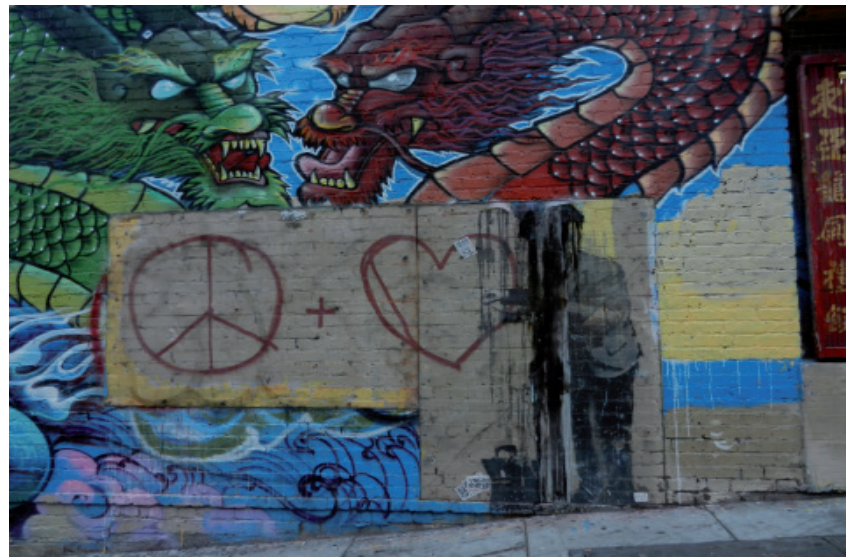

Figura 7. Peaceful Hearts de Banksy (Chinatown, San Francisco, CA). Protección con plexiglás, deterioro directo sobre la placa (2013), Fotografía Ana Lizeth Mata Delgado

Madona con niño creada en Alemania en la década de los años noventa. Esa obra fue descubierta en 2012 debajo de una serie de posters. Sin embargo, fue Maxi Kretzschmar, fue quien identificó y promovió que la obra fuese restaurada por un equipo de restauradores profesionales, incluso contaron con la participación del artista para ello. Curiosamente la obra no solo fue restaurada sino protegida de igual manera que los casos anteriores por un plexiglás para evitar que sea dañada de nuevo. Cabe señalar que le fue colocada una cédula informativa. (Schilling, 2012). [Fig. 8] Lo interesante en este caso, no es solo que la obra se haya considerado restaurar, sino que además fue realizada por profesionales en el campo con experiencia y por consecuencia quizá su intervención fue más apegada a los criterios usados en la pintura mural tradicional. Aunado a que el artista estuvo de acuerdo en la conservación de la misma. Este caso es relevante no solo por la intervención y los involucrados, sino porque además pasó a formar parte de la lista de obras relevantes de la ciudad, dando paso a la resignificación de la obra.

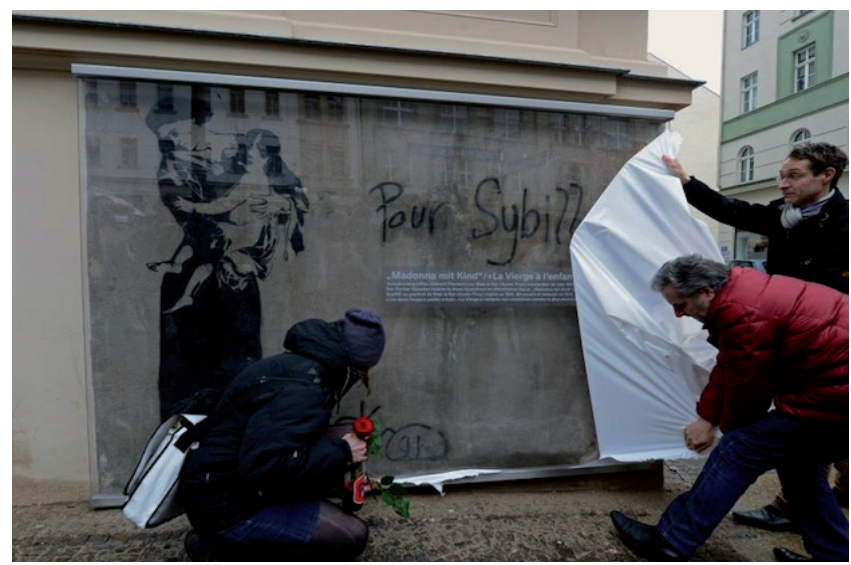

Figura 8. Blek Le Rat's «Madonna and Child» is back. photo: Hendrik Schmidt. Fotografía tomada de https://artsation.com/en/journal/ editorial/blek-le-rat-in-leipzig
Finalmente para dar cabida a las diversas alternativas de intervención, se considera la reposición como una opción si la obra se ha perdido por completo. Esta decisión debe de analizarse desde varias perspectivas pues es a partir de la relevancia de la obra, el productor, el contexto y el impacto que esta tiene en el entorno que la contiene e incluso la trascendencia como producción artística, es que se puede considerar como una alternativa viable.

Para ejemplificar esta alternativa se abordará el caso del mural Keith Haring pintó en Barcelona en el año de 1989 Todos juntos podemos parar el sida el cual realizó en el barrio del Raval, conocido entonces como el "Barrio Chino". Pese a la relevancia del artista para ese momento, la obra no tuvo un mantenimiento adecuado que promoviera su conservación y además el muro en donde fue creado sería demolido por la puesta en marcha Plan Especial de Reforma Interior del barrio del Raval. Debido a esta circunstancia a esta circunstancia, los responsables de obra de Haring y el Museo de Arte Contemporáneo de Barcelona (MACBA), en colaboración con el Ayuntamiento de Barcelona iniciaron el 1992 la calca de la obra y la toma de muestras del tipo de pintura con la que fue realizada para dar paso en un futuro a la reproducción de la misma.

Esta obra se ha reproducido en tres ocasiones (1996, 1998, 2014) en un muro de hormigón al exterior del MACBA, quedando a la vista de todos y si bien cambió de contexto, sigue cumpliendo con la función de transmitir un mensaje por demás relevante para la comunidad, pues esta obra no solo habla de su trabajo sino de la situación sanitaria que aquejaba al mundo en la década de los años ochenta, el impacto y relevancia que tuvo la presencia del artista y de la obra dentro de la ciudad y la trascendencia de la misma. (http://www.macba.cat/es/todos-juntos-podemos-pararel-sida-1465 [consultado 11/10/2016])

Es interesante analizar una opción como esta, pues si bien es una ganancia que la obra perdure y mantenga el diseño intacto e incluso se haya podido utilizar el mismo tipo de pintura para dar continuidad y autenticidad a la misma, el contexto en el que ahora se encuentra tiene un impacto diferente quizá en la significación de la obra. Para Haring la elección del sitio para pintarla no fue fortuito, eligió un sitio en donde diariamente aparecían jeringuillas y que le recordaban a los barrios de Nueva York, en donde además el muro donde fue realizado presentaba una inclinación importante que implicaba una adaptación anatómica por parte del artista para poder llevarla a cabo.

Ahora el espacio abierto en el que se encuentra la obra no tiene ninguna estas dos circunstancias, sin embargo lo importante aquí es la permanencia del mensaje a través de la reproducción del mural. [Fig. 9]

Es interesante cómo a pesar de que no se considera que este tipo de obras trasciendan o se conserven desde inicio, si hay una conciencia por parte de algunos artistas $y / o$ profesionales del campo de la restauración y afines, para que 


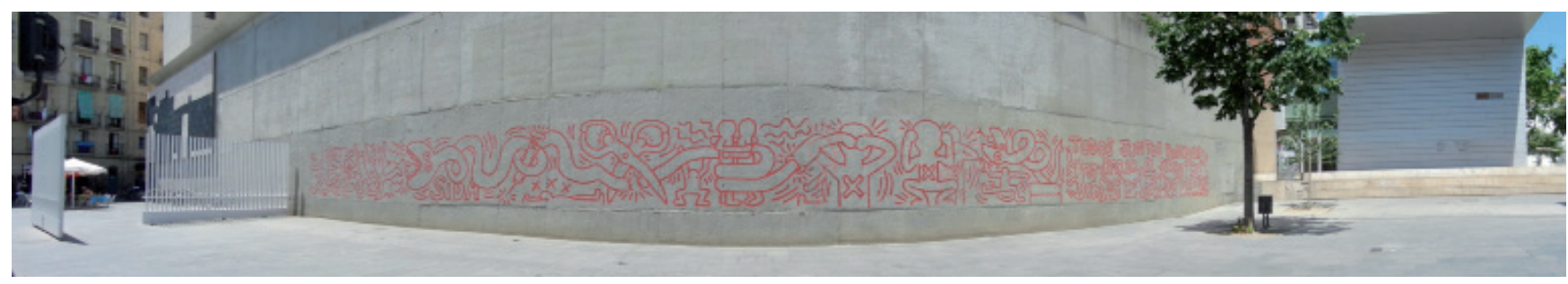

Figura 9. Todos juntos podemos para el sida, Keith Haring, 1989. Reproducción del mural explanada del MACBA (Barcelona, España). Fotografías Ana Lizeth Mata Delgado

la obra se mantenga en buen estado el mayor tiempo posible. En el caso de algunos artistas tienen contemplado desde el sitio donde la elaborarán hasta el material que usarán, cabe mencionar que actualmente existe una gama importante de materiales realizados exprofeso para la elaboración de arte urbano. "En lo que se refiere al Street Art y al Graffitti, la elección de materiales y el contexto donde se desarrollan juegan un papel fundamental para complementar su significado y el concepto que el artista proporcionó en un inicio, además de que en muchos casos no es una obra concluida sino que puede complementarse con diversas intervenciones de distintas temporalidades sino que pueden ser realizadas por otros artistas." (Mata 2013:3)

Incluso algunos consideran que esto puede ser necesario y fomentan su trascendencia, como lo comenta Abarca "alargar la vida de sus obras dentro de lo que las circunstancias lo permiten" (Abarca 2010: 90).

No obstante, es normal y derivativo que sea el entorno urbano el que plantee una serie de cambios y alteraciones sobre las obras, como bien comenta Anna Waclawek, esta interacción plantea una constante transformación que modifica y en algunos casos resignifica la obra.

"El proceso de nacimiento y muerte de una obra callejera en el contexto de la ciudad, atrae a muchos artistas por razones conceptuales y estructurales. [...] La experiencia [del Street Art] como un proceso transitorio está inextricablemente ligado al significado de la obra como un elemento de la composición cambiante de la ciudad. [...] La temporalidad del Street Art por lo tanto, traduce visualmente la experiencia de ser parte de la materia constitutiva de la ciudad." (Waclawek 2011: 91).

\section{Conclusiones}

Los posibles cruces entre la Teoría de la Restauración y el Arte Urbano aún no son del todo claros, pues dependerá del caso a tratar y de la manera en cómo sea abordado y por quién lo que determinará esta intersección. Sin embargo, es interesante como durante el análisis de las posibles alternativas y alcances que se tienen para conservar el arte urbano, podemos observar que no es una tarea ajena al campo disciplinar, por el contrario, cada vez los conservadores-restauradores se están involucrando a participar en la intervención de este tipo de manifestaciones artísticas e incluso a proponer alternativas para su conservación y/o restauración. Además de las intervenciones directas, cada vez más se desarrollan grupos de trabajo e investigación en torno al tema constituyéndose no solo por artistas y/o restauradores, sino en conjunto con otras disciplinas como la antropología, la sociología, la gestión o la curaduría por mencionar algunas, dando paso a nutridas discusiones en torno al tema.

En los casos mostrados para las cuatro alternativas de conservación propuestas, se plantea que en al menos dos de ellas ya sean restauradores profesionales los que se están involucrando en la ejecución de los procesos, incluso en algunos casos con la ayuda del artista como Blak le Rat en el caso de la Madona con el niño o en la reproducción de la obra de Keith Haring Todos juntos podemos parar el sida.

Es importante, tomar en cuenta que el arte urbano ha tenido que adaptarse a las nuevas tendencias no solo de su propio quehacer sino de aquellas disciplinas alrededor de ella en donde la perspectiva sobre su existencia ha cambiado, ya no solo se plantean la desaparición de inicio, sino que su proyección, aceptación, comprensión e incluso el interés que se tiene en ellas ha dado paso para tener una nueva perspectiva. Si bien, conservarlas y/o restaurarlas pareciera una afrenta directa a su propia naturaleza, también es cierto que no han tenido el suficiente espacio temporal para que se puedan visualizar de otra manera, no solo desde la perspectiva y relevancia del artista, sino desde la manufactura involucrada, la mezcla de materiales, el contexto, la importancia del tema o el impacto en la trasmisión del mensaje; sino como un documento que aportará información relevante al futuro sobre el tipo de manifestaciones artísticas desarrolladas en un periodo específico.

Una de las principales problemáticas a las que se enfrenta el conservador-restaurador es la diversidad material en la producción de arte urbano es por ello que resulta fundamental conocer y comprender los alcances y limitaciones de la disciplina ante este tipo de obras. De ahí también deriva el análisis sobre cuáles serían los criterios de intervención que tendrían que aplicarse para la conservación-restauración de estas obras.

Una alternativa que permite conservar la obra sin alterar su naturaleza efímera, procesual y transgresora como se 
mencionó antes, es quizá la documentación; puede ser una alternativa intermedia entre que desaparezcan y no quede ninguna referencia de la obra más que apelar a la memoria de quienes la conocieron versus la restauración de la misma, interviniendo cabalmente la materia que la conforma e inmortalizando aquello que fue creado para tener una existencia breve pero trascendental. Como lo comenta De la Rubia "A veces el punto medio ante discrepancias de este tipo se basará en tomar medidas plenamente preventivas, que ayuden a frenar el deterioro natural, pero sin intención de acabar con éste y asumiendo que la obra debe desaparecer" (De la Rubia 2013: 44).

En unos años podremos mirar al pasado y evaluar qué alternativa funcionó mejor, pero por lo pronto aprovechemos los nuevos recursos tecnológicos para documentar las obras, complementando idealmente con la opinión del artista a partir de realizar una entrevista con él (ella) para comprender adecuadamente cada una de las producciones artísticas vinculadas al arte urbano y hacer que nuestro proceder cualquiera que este sea, esté sustentado adecuadamente.

Una vez analizadas las diversas alternativas de conservación para el arte urbano, es pertinente e importante analizar de qué manera estamos asumiendo y comprendiendo la producción de arte urbano, ¿es la intervención directa, la manera correcta de conservarla? ¿Debemos dejar que la obra siga su curso, a pesar de que resulte relevante e importante para un público o una comunidad específicos? Quizá si buscáramos poner en balanza a la disciplina profesional de la restauración con el arte urbano, se podría pensar que el criterio usado más adecuado considerando sus características primarias es la "mínima intervención necesaria" no obstante, ¿qué tan válido es contener el proceso de degradación natural de la obra? ¿Es factible limitar su interacción con el contexto circundante e incluso el diálogo que establece de forma directa con las obras de otros artistas cercanos a fin de que se mantenga en las mejores condiciones posibles? Estos y otros cuestionamientos surgirán mientras no se determine claramente hasta donde es factible conservar y/o restaurar de esta naturaleza sin contrarrestar su origen e intención.

Para finalizar me parece importante plantear que la aplicación de criterios, se enfocará más en el planteamiento teórico y ético del hecho en sí mismo de restaurar o no, que en la manera de llevarlo a cabo, pues quizá los procesos técnicos no cambien radicalmente en la ejecución, sino más bien es la toma de decisión, la metodología planteada y los alcances de nuestra interacción con la obra desde el punto de vista de la disciplina.

\section{Bibliografia:}

ABARCA S. F. J. (2010). El postgraffiti, su escenario y sus raíces: graffiti, punk, skate y contrapublicidad, Tesis de Doctorado. Madrid: Facultad de Bellas Artes de la Universidad Complutense de Madrid.
ARROYO, S. R. (2015). CODEX Una aproximación al grafiti de la Ciudad de México. México: CONACULTA, TURNER.

MARCIAL, R. (2012)“El graffiti como discurso gráfico en la disidencia juvenil" en Pura imagen. Sarah Corona Berkin (coord.). México: CONACULTA

WACLAWEK, A. (2011). Graffiti and Street Art. Londres: Thames \& Hudson.

\section{Vinculos web}

DE LA RUBIA LÓPEZ, E. (2013). Arte urbano: graffiti y postgraffiti. Acercamiento a la problemática legal y patrimonial en torno a su conservación.TesisFinaldeMásterenConservacióny Restauraciónde Bienes Culturales, Universidad Politécnica de Valencia. Dirigida por Rosario Llamas Pacheco y Mercedes Sánchez Pons. PDF disponible en: http://riunet.upv.es/bitstream/handle/10251/39193/TESIS\%20 FINAL.pdf?sequence=1\&isAllowed $=y$ [consulta: 20/08/2016].

MATA DELGADO A. L., (2013) “Conservando el Street Art \& Graffiti, la pertinencia de su conservación y la problemática material derivada de su técnica de manufactura" en Memorias del X Foro Académico ECRO. Documento electrónico disponible en http://www.ecro.edu. mx/memorias_x_foro.html [consulta: 20/08/2016]

MATA DELGADO A. L. (2016). "Cartografías del arte urbano: su localización como medio de difusión, registro y conservación" en Archivo Churubusco. México: ENCRyM-INAH http:// archivochurubusco.encrym.edu.mx/cartografia.html [consultado 25/09/2016]

PHILIPOT, P. (2015). "La obra de arte, el tiempo y la restauración" en Conversaciones con Paul Phillipot. Revista de Conservación. México: CNCPC-INAH http://conservacion.inah.gob.mx/ publicaciones/?cat=350 [consultada 26/09/2016]

SCHILLING, J. (2012). "Preserving art that was never meant to last", Deutsche Welle, 7 de mayo de 2012. Disponible en: http://www. dw.de/preserving-art-that-was-never-meant-to-last/a-15933463 [consulta 10]/09/2016].

http://cultura.elpais.com/cultura/2013/06/03/ actualidad/1370285346_249416.html [consultado 9/09/2016]

http://scottreither.com/blogwp/2012/10/18/dear-banksy/ [consultado 25/09/2016]

http://warholian.com/2010/04/banksy-defaced-in-chinatownwarholian-san-francisco/ [consultado 25/09/2016]

https://artsation.com/en/journal/editorial/blek-le-rat-in-leipzig [consultado 20/09/2016]

https://www.youtube.com/watch?v=T-sEg_b3Fic [consultado 23/09/2017]

http://www.macba.cat/es/todos-juntos-podemos-parar-elsida-1465 [consultado 23/09/2016] 
http://ccaa.elpais.com/ccaa/2014/02/27/

catalunya/1393535246_601176.html [consultado 11/10/2016]

h t t p: / / c caa.elpais.com/ccaa/2014/02/2 7 /

catalunya/1393535246_601176.html [consultada el 12/10/2016]

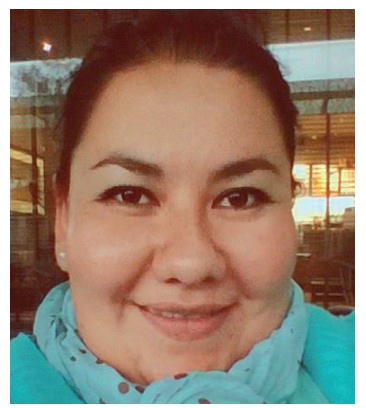

\author{
Ana Lizeth Mata Delgado \\ ENCRyM-INAH \\ lizeth_matadelgado@yahoo.com.mx
}

Licenciada en Restauración de Bienes Muebles por la Escuela Nacional de Conservación, Restauración y Museografía-INAH. Profesora titular del Seminario Taller de Restauración de Obra Moderna y Contemporánea en la ENCRyM-INAH. Maestrante de Historia del Arte por la Universidad Nacional Autónoma de México con especialidad en Arte Contemporáneo. Ponente en diversos foros de especialidad nacionales e internacionales (EUA, Argentina, Chile, España) en conservación-restauración de arte contemporáneo y arte urbano. Organizadora de los Cursos de Restauración de Arte Moderno y Contemporáneo llevados a cabo en la ENCRyM en colaboración con el MoMA de Nueva York en 2008 y 2010, respectivamente. En 2011 y 2013 impartió y coordinó el Curso Introductorio a la Restauración de Arte Moderno y Contemporáneo para el personal del CENCROPAM-INBA. Co-coordinó el 1er. Encuentro de INCCA (International Network for Conservation of Contemporary Art) en México, la conformación de la Red Iberoamericana. El Encuentro de Conservación de Street Art y Graffiti en 2013 y el Encuentro Estrategias de Conservación en Arte Contemporáneo en colaboración con el MUAC-UNAM y Fundación Jumex Arte Contemporáneo en 2014. En 2015 coordinó e impartió el curso “Una década conservando el arte contemporáneo" en colaboración con el Museo de Arte Contemporáneo de Barcelona y las conferencias "Entre restauradores te veas. Charlando con artistas contemporáneos sobre restauración". En ese mismo año llevó a cabo una estancia de investigación en torno a la conservación de arte contemporáneo en el Museo Nacional Centro de Arte Reina Sofía en Madrid, España.

Es miembro del International Network for Conservation of Contemporary Art, del Grupo de Arte Urbano del Grupo Español del IIC y de la Red Iberoamericana de Restauración de Arte Contemporáneo. 